\title{
FLATNESS BASED TRAJECTORY GENERATION OF QUANTUM SYSTEMS
}

\author{
Pierre Rouchon
}

\author{
Ecole des Mines de Paris, Centre Automatique et \\ Systèmes, 60 Bd Saint-Michel, 75272 Paris cedex 06 \\ FRANCE.Email: pierre.rouchon@ensmp.fr \\ NOLCOS 2004
}

\begin{abstract}
A two-states quantum system with one control is proved to be flat. This provides a simple procedure to design smooth open-loop controls that steer in finite time from one eigen-state to the other one. A three-states quantum system with one control is not flat in general. Following the Rabi oscillations used by physicists to control stimulated transition, we associate to this system an averaged control system where the number of controls is increased and where flatness-based motion planning techniques can be used. This allows to steer directly from one eigen-state to any other one without using an additional intermediate eigen-state. In certain energy configurations our method is a noticeable improvement of the standard Rabi transitions strategy and leads to " active tunnelling control", as illustrated by simulations. This method can be extended without major difficulties to higher dimensional cases.
\end{abstract}

Keywords: Quantum oscillators, controllability, averaging, motion planning, flatness based control, trajectory generation.

\section{INTRODUCTION}

The control of quantum system is an active subject of fundamental and practical importance in chemistry (see, e.g., (Levis et al., 2001)). It appears that the controllability of quantum systems described by a finite number of states is quite well understood (see, e.g. (Ramakrishna et al., 1995; Turinici, 2000b; Turinici, 2000a)). However there is still a need to have simple control design method. In (Vettori, 2002; Ferrante et al., 2002; Mirrahimi and Rouchon, 2004a; Mirrahimi and Rouchon, 2004b) Lyapounov based methods are proposed. This paper proposes flatness based methods (Fliess et al., 1995; Fliess et $a l ., 1999)$ for steering from a pure state to another pure state. The main advantage of such method is its computational simplicity and its simple interpretation in physical terms.
We consider quantum systems described by the following Schrödinger equation

$$
\imath \hbar \dot{\psi}=\left(H_{0}+u H_{1}\right) \psi
$$

where $\psi$ is the complex probability amplitude vector (belongs to an Hilbert space), $H_{0}$ is the free Hamiltonian and $H_{1}$ is the Hamiltonian associated to the scalar control $u$ (corresponding to a classical electric field generated by a laser). We focus here on the following motion planning problem: for two pure states, $\psi_{a}$ and $\psi_{b}$ of free energy $E_{b}$ and $E_{b}\left(H_{0} \psi_{a}=E_{a} \psi_{a}\right.$ and $\left.H_{0} \psi_{b}=E_{b} \psi_{b}\right)$, find an open-loop control $[0, T] \ni t \mapsto u(t)$ steering the state $\psi$ form $\psi_{a}$ at $t=0$ to the state $\psi_{b}$ at $t=T>0$.

We first consider a two-state system $\left(\psi \in \mathbb{C}^{2}\right)$ that is proved to be flat for almost all Hamiltonians $H_{0}$ and $H_{1}$. The flat output admits a clear physical 
interpretation when the Bloch sphere model is used. The second class of systems we consider has three states $\psi \in \mathbb{C}^{3}$ and a special structure of $H_{0}$ and $H_{1}$ as illustrated on figure 1 . Such structure renders one particular transition between two eigen-states not so easy (two-photons transition). Such transition requires, when Rabi oscillations are used, to pass via the remaining third eigenstate. Our method avoids this intermediate step. The steering control is then obtained by considering, under the classical weak field approximation, the average dynamics that admits then two control variables. Flatness-based motion planning techniques provide easily the steering control for the averaged dynamics (see (Fliess et al., 1995) where a similar method is used to control the Kapitsa pendulum). Simulations show that such approximate steering control remains quite attractive since the amplitude of the control does not need to be very small to reach the final state.

Preliminary version of these results can be found in (Rouchon, 2002).

\section{TWO STATES SYSTEMS}

Consider now a two states system. Its wave function $\psi$ belongs to $\mathbb{C}^{2}$. The Schrödinger equation

$$
\imath \hbar \dot{\psi}=\left(H_{0}+u H_{1}\right) \psi
$$

involves the $2 \times 2$ Hermitean matrices $H_{0}$ and $H_{1}$ :

$$
H_{0}=\left(\begin{array}{cc}
-E / 2 & 0 \\
0 & E / 2
\end{array}\right), \quad H_{1}=\left(\begin{array}{cc}
h_{1} & b \\
b^{*} & h_{2}
\end{array}\right)
$$

with $E, h_{1}, h_{2} \in \mathbb{R}$ and $b \in \mathbb{C}^{*}$. With $\psi=$ $\left(a_{1}, a_{2}\right) \in \mathbb{C}^{2}$, the density matrix is defined by

$$
\rho=|\phi\rangle\langle\phi|=\left(\begin{array}{cc}
\left|a_{1}\right|^{2} & a_{1}^{*} a_{2} \\
a_{1} a_{2}^{*} & \left|a_{2}\right|^{2}
\end{array}\right) .
$$

In terms of Pauli matrices

$$
\sigma_{x}=\left(\begin{array}{ll}
0 & 1 \\
1 & 0
\end{array}\right), \quad \sigma_{y}=\left(\begin{array}{cc}
0 & -\imath \\
\imath & 0
\end{array}\right), \quad \sigma_{z}=\left(\begin{array}{cc}
1 & 0 \\
0 & -1
\end{array}\right),
$$

it reads

$$
\rho=1+\lambda \sigma_{x}+\mu \sigma_{y}+\nu \sigma_{z}
$$

with $\boldsymbol{S}=(\lambda, \mu, \nu) \in \mathbb{S}^{2}$. This corresponds to a classical change of coordinates (Bloch sphere model, see (Cohen-Tannoudji et al., 1977; Abragam, 1961)) where the meaningless absolute phase is removed: $\phi=\left(a_{1}, a_{2}\right) \in \mathbb{C}^{2} /\{0\}$ and $\tilde{\phi}\left(\tilde{a}_{1}, \tilde{a}_{2}\right) \in$ $\mathbb{C}^{2} /\{0\}$ are the probabilities amplitudes of the same physical state if and only if exists $\theta \in \mathbb{S}^{1}$ such that $a=\exp (\imath \theta) \tilde{a}$. Notice that $\boldsymbol{S} \in \mathbb{S}^{2}$ comes from $\left|a_{1}\right|^{2}+\left|a_{2}\right|^{2}=1$. In the Bloch coordinates the dynamics reads

$$
\dot{\boldsymbol{S}}=\boldsymbol{S} \wedge\left(\omega_{0} \boldsymbol{B}_{0}+\frac{u}{\hbar} \boldsymbol{B}_{1}\right)
$$

where $\omega_{0}=E / \hbar$ and

$$
\boldsymbol{B}_{0}=\left(\begin{array}{l}
0 \\
0 \\
1
\end{array}\right), \quad \boldsymbol{B}_{1}=\left(\begin{array}{c}
-2 \Re(b) \\
2 \Im(b) \\
h_{1}-h_{2}
\end{array}\right) .
$$

Set $\tau=\omega_{0} t,{ }^{\prime}=d / d \tau$ and $v=\frac{\left\|\boldsymbol{B}_{1}\right\|}{\omega_{0} \hbar} u$ the new control. The dynamics becomes

$$
\boldsymbol{S}^{\prime}=\boldsymbol{S} \wedge\left(\boldsymbol{B}_{0}+v \boldsymbol{J}\right)
$$

where $\boldsymbol{J}$ is the unitary vector $\frac{1}{\left\|\boldsymbol{B}_{1}\right\|} \boldsymbol{B}_{1}$. Denote by $\alpha \in] 0, \pi\left[\right.$ the angle between $\boldsymbol{B}_{0}$ and $\boldsymbol{J}$ and consider the ortho-normal frame $(\boldsymbol{I}, \boldsymbol{J}, \boldsymbol{K})$ with $\boldsymbol{K}=\boldsymbol{B}_{0} \wedge \boldsymbol{J} / \sin \alpha$ and $\boldsymbol{I}=\boldsymbol{J} \wedge \boldsymbol{K}$. Set $\boldsymbol{S}=x \boldsymbol{I}+$ $y \boldsymbol{J}+z \boldsymbol{K}\left((x, y, z) \in \mathbb{R}^{3}\right.$ with $\left.x^{2}+y^{2}+z^{2}=1\right)$. Then the dynamics reads

$$
\begin{aligned}
& x^{\prime}=-z(\cos \alpha+u) \\
& y^{\prime}=z \sin \alpha \\
& z^{\prime}=x(\cos \alpha+u)-y \sin \alpha
\end{aligned}
$$

since $\boldsymbol{B}_{0}=\sin \alpha \boldsymbol{I}+\cos \alpha \boldsymbol{J}$. Notice that $x^{2}+y^{2}+z^{2}$ is invariant. The restriction of the dynamics on $\mathbb{S}^{2}$ is flat with $y$ as flat output:

$$
z=y^{\prime} / \sin \alpha, \quad x= \pm \sqrt{1-y^{2}-\left(y^{\prime}\right)^{2} / \sin ^{2} \alpha} .
$$

Let us now find a smooth control $[0, T] \ni t \mapsto$ $u(t), u(0)=0$ and $u(T)=0$, steering from $-E / 2$ to $+E / 2$. When $u=0$, the state of energy $-E / 2$ corresponds, in the Bloch-coordinates, to $(x, y, z)=(-\sin \alpha,-\cos \alpha, 0)$ and the state of energy $+E / 2$ to $(x, y, z)=(\sin \alpha, \cos \alpha, 0)$.

Assume to simplify that $\alpha=\pi / 2$ (a similar construction exists for over values of $\alpha$ ). Set $p(\tau)=(1-\tau) \tau^{2}(2-\tau)^{2}$. Simple computations show that the function $f(\tau)=1-p^{2}(\tau)-\left(p^{\prime}\right)^{2}(\tau)$ is non negative on $[0,2]$, reaches 0 only for $\tau=1$ with $f^{\prime \prime}(1)>0$. Thus the function $g: \mathbb{R} \mapsto \mathbb{R}$ defined by

$$
g(\tau)= \begin{cases}-1 & \text { if } \tau<0 \\ -\sqrt{f(\tau)} & \text { if } \tau \in[0,1] \\ \sqrt{f(\tau)} & \text { if } \tau \in[1,2] \\ 1 & \text { if } \tau>2\end{cases}
$$

is $C^{2}$. The control

$$
v(\tau)=-g^{\prime}(\tau) / p^{\prime}(\tau)
$$

is well defined even for $\tau$ around 1 . It steers the system from $(-1,0,0)$ at $\tau=0$ to $(1,0,0)$ at $\tau=2$. The steering trajectory is

$$
x(\tau)=g(\tau), \quad y(\tau)=p(\tau), \quad z(\tau)=p^{\prime}(\tau) .
$$

The interest of the above computations relies on the fact that the control $u$ is smooth. This is not the case for standard steering control of $\pm 1 / 2$ spin systems (Abragam, 1961): $u$ is then piecewise constant and discontinuous; the steering trajectory is made of Larmor precessions over finite time interval.

\section{THREE STATES SYSTEM}

Consider now a three states system with three energy levels $E_{1}<E_{2}<E_{3}$ associated to the 


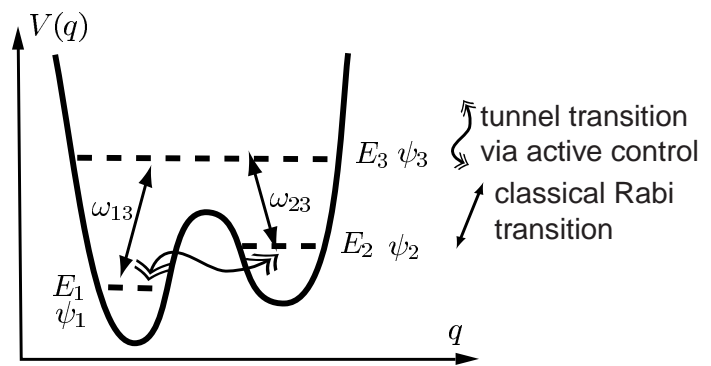

Fig. 1. A three states system: steering from $E_{1}$ to $E_{2}$ without reaching $E_{3}$ via active tunnelling control.

physical case illustrated on figure 1 . It corresponds to the reduced model of a $1 D$ particule in the potential $V(q)$ admitting two minima with bounded states $\psi_{1}$ and $\psi_{2}$ of low energy $E_{1}$ and $E_{2}$ separated by a potential barrier and a third bounded state $\psi_{3}$ of energy $E_{3}$ passing over the barrier. The supports of the $\psi_{i}, i=1,2,3$ are roughly sketched by the dashed horizontal lines. The three states model is a finite dimensional approximation of $\left(p=\imath / \hbar \frac{\partial}{\partial q}\right)$

$$
1 \hbar \dot{\psi}=\left(p^{2} / 2 m+V(q)-u q\right) \psi
$$

then entries $(1,2)$ and $(2,1)$ in $H_{1}$ are negligible. We have the following dynamics

$$
\begin{aligned}
\imath \hbar \dot{\alpha}_{1} & =\left(E_{1}+u\langle q\rangle_{1}\right) \alpha_{1}+u\langle q\rangle_{13} \alpha_{3} \\
\imath \hbar \dot{\alpha}_{2} & =\left(E_{2}+u\langle q\rangle_{2}\right) \alpha_{2}+u\langle q\rangle_{23} \alpha_{3} \\
\imath \hbar \dot{\alpha}_{3} & =\left(E_{3}+u\langle q\rangle_{3}\right) \alpha_{3}+u\langle q\rangle_{31}^{*} \alpha_{1}+u\langle q\rangle_{32}^{*} \alpha_{2}
\end{aligned}
$$

where

$$
\langle q\rangle_{i}=\int\left|\psi_{i}\right|^{2} q d q, \quad\langle q\rangle_{i j}=\int \psi_{i}^{*} \psi_{j} q d q
$$

and $\psi=\alpha_{1} \psi_{1}+\alpha_{2} \psi_{2}+\alpha_{3} \psi_{3}$ with $a_{i} \in \mathbb{C}$. Change the phase as follows for $i=1,2,3$ :

$$
\alpha_{i}=\exp \left(-\imath E_{3} / \hbar t-\int_{0}^{t} u(s)\langle q\rangle_{3} / \hbar d s\right) a_{i} .
$$

Then

$$
\begin{aligned}
\imath \dot{a}_{1} & =\left(\omega_{13}+u e_{1}\right) a_{1}+u b_{1} a_{3} \\
\imath \dot{a}_{2} & =\left(\omega_{23}+u e_{2}\right) a_{2}+u b_{2} a_{3} \\
\imath \dot{a}_{3} & =u b_{1}^{*} a_{1}+u b_{2}^{*} a_{2}
\end{aligned}
$$

where $\omega_{13}=\left(E_{1}-E_{3}\right) / \hbar$ and $\omega_{23}=\left(E_{2}-E_{3}\right) / \hbar$ are the Bohr frequencies, $e_{i}=\left(\langle q\rangle_{i}-\langle q\rangle_{3}\right) / \hbar$, $b_{1}=\langle q\rangle_{13} / \hbar$ and $b_{2}=\langle q\rangle_{23} / \hbar$.

Finding explicit open-loop control $[0, T] \ni t \mapsto$ $u(t)$ steering from the pure state of energy $E_{1}$, $a=(1,0,0)$, to the pure state $E_{2}, a=(0,1,0)$ without passing via the intermediate state $E_{3}$ is not so obvious. We propose here an open-loop design mixing standard perturbation techniques (see, e.g., (Messiah, 1962)) and flatness based steering methods.

Assume that the control $u$ is small, $\left|u b_{i}\right|,\left|u e_{j}\right| \ll$ $\omega_{13}, \omega_{23}$ and varies slowly (time constant much smaller than $T_{23}=2 \pi / \omega_{23}$ and $T_{13}=2 \pi / \omega_{13}$ the Bohr periods). Set $b_{1}=r_{1} \exp \left(\imath \theta_{1}\right), b_{2}=$ $r_{2} \exp \left(\imath \theta_{2}\right)$ with $r_{i}>0$ and $\theta_{i}$ real. Set

$$
u=\frac{2 v_{1}(t)}{r_{1}} \cos \left(\omega_{13} t\right)+\frac{2 v_{2}(t)}{r_{2}} \cos \left(\omega_{23} t\right)
$$

with $v_{1}$ and $v_{2}$ small amplitude. Then classical averaging techniques show that, when $\omega_{13} / \omega_{23}$ is irrational, the solutions of (2) are close to the solution of the average system

$$
\begin{aligned}
& \dot{x}_{1}=v_{1} x_{3} \\
& \dot{x}_{2}=v_{2} x_{3} \\
& \dot{x}_{3}=-v_{1} x_{1}-v_{2} x_{2}
\end{aligned}
$$

where

$$
\begin{aligned}
& a_{1}=\exp \left(\imath\left(\theta_{1}-\omega_{13} t\right)\right) x_{1} \\
& a_{2}=\exp \left(\imath\left(\theta_{2}-\omega_{23} t\right)\right) x_{2} \\
& a_{3}=\imath x_{3} .
\end{aligned}
$$

When one of the $v_{i}$ is constant and the other one is zero we recover the classical Rabi oscillations (Messiah, 1962). They can be used to steer, in a first step, the state from energy $E_{1}$ to energy $E_{3}$ with $v_{1}$ constant $\neq 0$ and $v_{2}=0$ and then, in a second step, to steer the system from $E_{3}$ to $E_{2}$ with $v_{1}=0$ and $v_{2}$ constant $\neq 0$. We will see that we can mix these two steps to steer directly the system from $E_{1}$ to $E_{2}$ without reaching the energy $E_{3}$.

Up to phase shifts that are not important from physical reasons, we have to find $v_{1}$ and $v_{2}$ steering the state $x$ from $(1,0,0)$ to $(0,1,0)$. Thus we can suppose that the components of $x$ remain real during the motion (since $u$ must be real, $v_{1}$ and $v_{2}$ are also real). Conservation of probability means that $I=x_{1}^{2}+x_{2}^{2}+x_{3}^{2}$ is invariant and equal to 1 and we have (with the positive branch $\left.x_{3}=\sqrt{1-x_{1}^{2}-x_{2}^{2}}\right)$ :

$$
\dot{x}_{1}=v_{1} \sqrt{1-x_{1}^{2}-x_{2}^{2}}, \quad \dot{x}_{2}=v_{2} \sqrt{1-x_{1}^{2}-x_{2}^{2}}
$$

with $x_{1}$ and $x_{2}$ as flat output. Take any increasing smooth bijection $s \mapsto \sigma(s)$ from $[0,1]$ to $[0,1]$ with

$$
\sigma(0)=0, \sigma(1)=1, \frac{d^{i} \sigma}{d s^{i}}(0)=\frac{d^{i} \sigma}{d s^{i}}(1)=0
$$

for $i=1,2,3$. Set $x_{1}=1-\sigma(t / T)$ and $x_{2}=$ $\sigma(t / T)$. Then the control

$$
\begin{aligned}
& v_{1}(t)=\frac{-\sigma^{\prime}(t / T)}{T \sqrt{2 \sigma(t / T)(1-\sigma(t / T))}} \\
& v_{2}(t)=\frac{\sigma^{\prime}(t / T)}{T \sqrt{2 \sigma(t / T)(1-\sigma(t / T))}}
\end{aligned}
$$

is well defined for $t \in[0, T]$, is smooth and satisfies $v_{i}(0)=v_{i}(T)=0, i=1,2$. Moreover it steers the average state $x$ from $(1,0,0)$ at $t=0$ to $(0,1,0)$ at $t=T$. Notice that when $T \gg T_{13}, T_{23}$, we automatically satisfy the averaging assumptions.

The simulations here below are based on model (2) with $\sigma$ a polynomial of degree 7 . We have considered three different cases: 

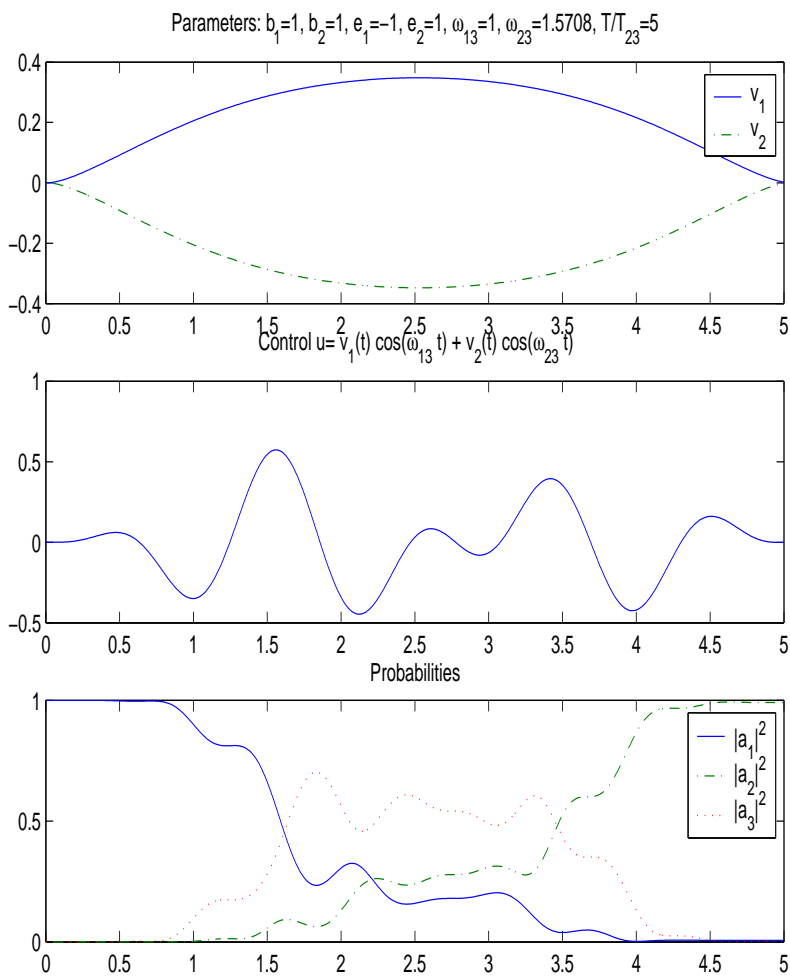

Fig. 2. Active tunneling from $E_{1}$ to $E_{2}$ without reaching $E_{3}>E_{1}, E_{2}$; the standard case.

- the standard case of figure 2 : the $b_{i}$ and $e_{i}$ are closed to one; the ratio of the Bohr frequency is irrational.

- the resonant case of figure 3: the ratio of the Bohr frequency is rational $\omega_{23}=2 \omega_{13}$.

- the ill conditioned case of figure 4: the system is close to a non controllable one; $\omega_{23} \approx \omega_{13}$. This case requires larger transition times $T$.

\section{GENERALIZATION}

Such control designs can be extended to quantum oscillators with more than 3 states. Such an extension relies on non-resonance assumptions, averaging techniques and the fact that the controllable part of the averaged system is flat.

Assume that $H_{0} / \hbar$ in (1) is the diagonal matrix $\operatorname{diag}\left(\omega_{k}\right)$, with $\omega_{k} \in \mathbb{R}$ for $k=1, \ldots, n, n$ being the dimension of the system. Denote by $b_{k, l} \in \mathbb{C}$ the entries of $H_{1} / \hbar$. The goal is to steer the system from the first pure state $(1,0, \ldots, 0)$ to the last one $(0, \ldots, 0,1)$. Assume that exists a path $\left(l_{1}, \ldots, l_{\sigma}\right) \in\{1, \ldots, n\}^{\sigma}$ of length $\sigma \geq 2$ such that, $l_{k} \neq l_{r}$ for $k \neq r, l_{1}=1, l_{\sigma}=n$ and for each $r \in\{1, \ldots, \sigma-1\}, b_{l_{r}, l_{r+1}} \neq 0$. Set

$$
u=\sum_{r=1}^{\sigma-1} u_{r}\left(e^{\imath\left(\omega_{l_{r}}-\omega_{l_{r+1}}\right) t}+e^{-\imath\left(\omega_{l_{r}}-\omega_{l_{r+1}}\right) t}\right)
$$

where $u_{r}$ are new controls. With $\psi=\exp \left(-\imath H_{0} t / \hbar\right) \phi$, (1) becomes
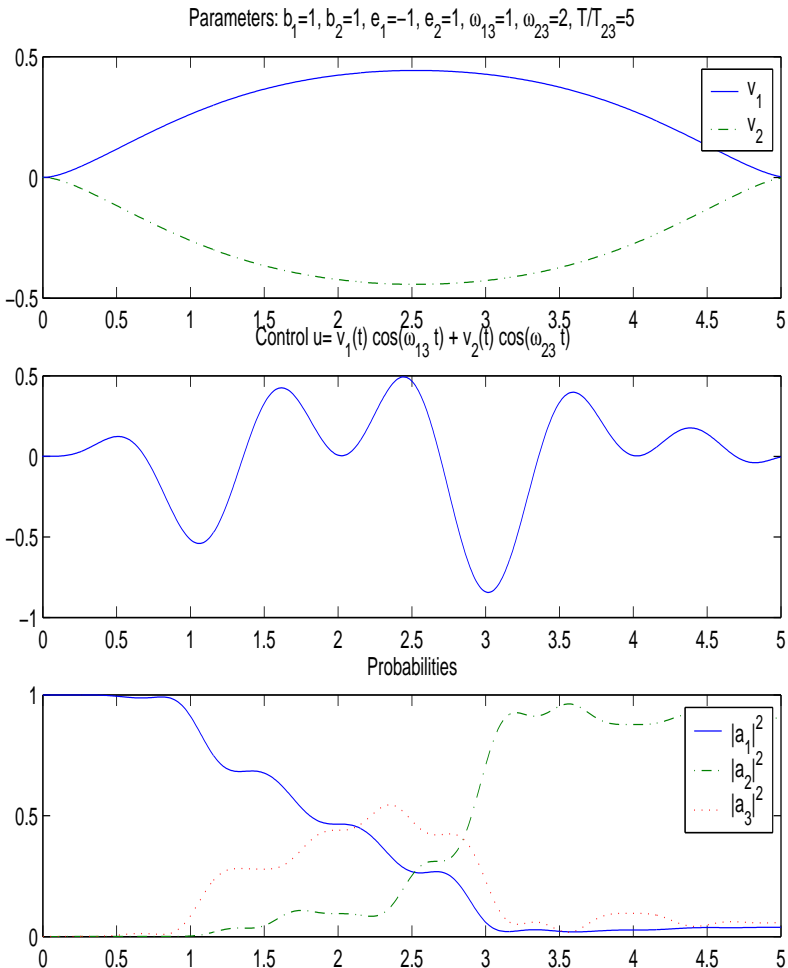

Fig. 3. Active tunneling from $E_{1}$ to $E_{2}$ without reaching $E_{3}>E_{1}, E_{2}$; the resonant case $E_{3}-$ $E_{2}=2\left(E_{3}-E_{1}\right)$.
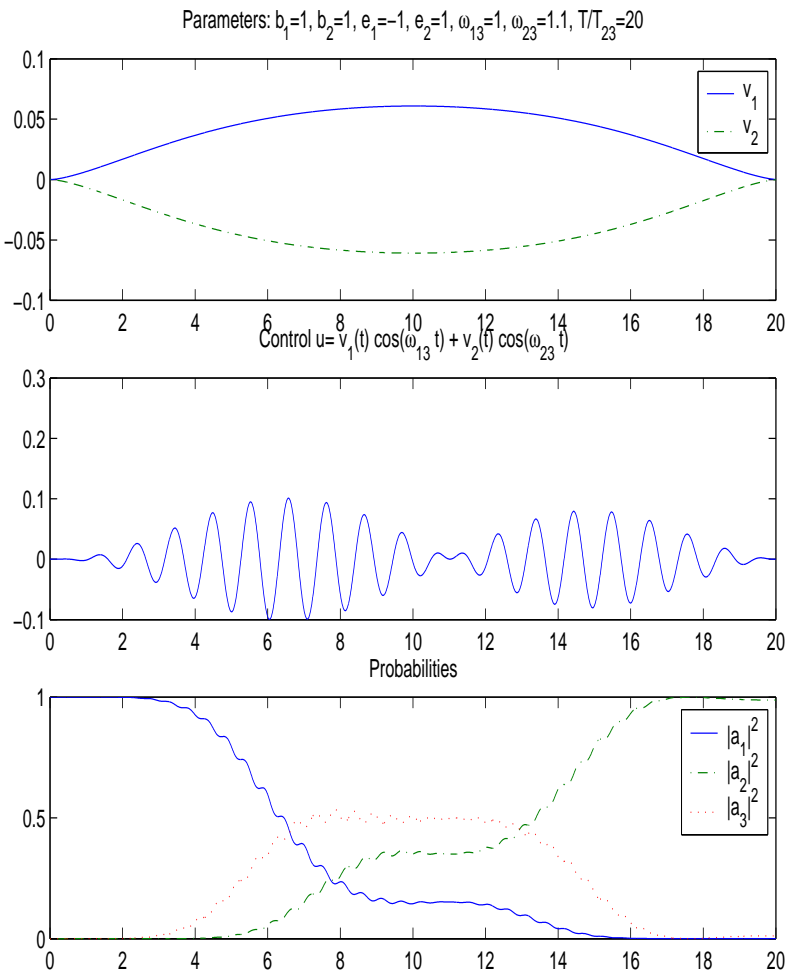

Fig. 4. Active tunneling from $E_{1}$ to $E_{2}$ without reaching $E_{3}>E_{1}, E_{2}$; the ill conditioned case $E_{3}-E_{2} \approx\left(E_{3}-E_{1}\right.$. 


$$
\begin{aligned}
\imath \dot{\phi}_{k}= & \sum_{r=1}^{\sigma-1} \sum_{s=1}^{n} u_{r} b_{k, s} \phi_{s} e^{\imath\left(\omega_{k}-\omega_{s}\right) t} \ldots \\
& \quad \ldots\left(e^{\imath\left(\omega_{l_{r}}-\omega_{l_{r+1}}\right) t}++e^{-\imath\left(\omega_{l_{r}}-\omega_{l_{r+1}}\right) t}\right) .
\end{aligned}
$$

Assume that $\left|\omega_{k}-\omega_{s}\right|=\left|\omega_{l_{r}}-\omega_{l_{r+1}}\right|$, implies $(k, s)=\left(l_{r}, l_{r+1}\right)$ or $(s, k)=\left(l_{r}, l_{r+1}\right)$. For $u_{r}$ small enough we can use the standard rotating wave approximation (called also secular approximation) and consider the following approximate average dynamics where the relative phases (the coherences) between the state components are lost:

$$
\begin{aligned}
\imath \dot{\phi}_{k} & =0 \quad \text { for } k \notin\left\{l_{1}, \ldots, l_{\sigma}\right\} \\
\imath \dot{\phi}_{l_{1}} & =u_{1} b_{l_{1}, l_{2}} \phi_{l_{2}} \\
& \vdots \\
\dot{\phi}_{l_{r}} & =u_{r} b_{l_{r}, l_{r-1}} \phi_{l_{r-1}}+u_{r+1} b_{l_{r}, l_{r+1}} \phi_{l_{r+1}} \\
& \vdots \\
\imath \dot{\phi}_{l_{\sigma}} & =u_{\sigma-1} b_{l_{\sigma}, l_{\sigma-1}} \phi_{l_{\sigma-1}}
\end{aligned}
$$

The components that do not belong to the transition path remain constant. Set $b_{l_{r}, l_{r+1}}=$ $c_{r} \exp \left(\imath \theta_{k}\right)$ with $c_{r}>0$ and $\theta_{r} \in \mathbb{R}$. Set $v_{r}=$ $c_{r} u_{r}$ and consider the following relative change of phases:

$$
\begin{aligned}
\phi_{l_{1}} & =x_{1} \\
\phi_{l_{2}} & =\imath e^{-\imath \theta_{1}} x_{2} \\
& \vdots \\
\phi_{l_{r}} & =(\imath)^{r-1} e^{-\imath\left(\theta_{1}+\ldots+\theta_{r-1}\right)} x_{r} \\
& \vdots \\
\phi_{l_{\sigma}} & =(\imath)^{\sigma-1} e^{-\imath\left(\theta_{1}+\ldots+\theta_{\sigma-1}\right)} x_{\sigma} .
\end{aligned}
$$

In the variables $x=\left(x_{1}, \ldots, x_{\sigma}\right)$, the approximate dynamics reads:

$$
\begin{aligned}
\dot{x}_{1} & =v_{1} x_{2} \\
\dot{x}_{2} & =-v_{1} x_{1}+v_{2} x_{3} \\
\vdots & \\
\dot{x}_{\sigma-1} & =-v_{\sigma-2} x_{\sigma-2}+v_{\sigma-1} x_{\sigma} \\
\dot{x}_{\sigma-2} & =-v_{\sigma-1} x_{\sigma-1} .
\end{aligned}
$$

We consider now only real values for $x$ (remember that the coherences are lost due to the rotating wave approximation). Notice that $x_{1}^{2}+\ldots+x_{\sigma}^{2}$ is an invariant (conservation of probability). The above system lives thus on the unit sphere of $\mathbb{R}^{\sigma}$. It is obviously controllable since it has $\sigma-1$ independent controls $\left(v_{1}, \ldots, v_{\sigma-1}\right)$. It is trivially flat with $x$ as flat output.

\section{REFERENCES}

Abragam, A. (1961). Principles of Nuclear Magnetism. Oxford University Press.
Cohen-Tannoudji, C., B. Diu and F. Laloë (1977). Mècanique Quantique. Vol. I\& II. Hermann, Paris.

Ferrante, A., M. Pavon and G. Raccanelli (2002). Control of quantum systems using modelbased feedback strategies. In: Fifteenth International Symposium on Mathematical Theory of Networks and Systems (MTNS).

Fliess, M., J. Lévine, Ph. Martin and P. Rouchon (1995). Flatness and defect of nonlinear systems: introductory theory and examples. Int. J. Control 61(6), 1327-1361.

Fliess, M., J. Lévine, Ph. Martin and P. Rouchon (1999). A Lie-Bäcklund approach to equivalence and flatness of nonlinear systems. IEEE AC 44, 922-937.

Levis, R.J., G.M. Menkir and H. Rabitz (2001). Selective bond dissociation and rearrangement with optimally tailored, strong-field laser pulses. Science 292, 709-713.

Messiah, A. (1962). Quantum Mechanics. Vol. I\&II. North Holland Publ. Co.. Amsterdam.

Mirrahimi, M. and P. Rouchon (2004a). Trajectory generation for quantum systems based on lyapounov techniques. In: NOLCOS.

Mirrahimi, M. and P. Rouchon (2004b). Trajectory tracking for quantum systems: a lyapounov approach.. In: MTNS.

Ramakrishna, V., M. Salapaka, M. Dahleh and H. Rabitz (1995). Controllability of molecular systems. Phys. Rev. A 51(2), 960-966.

Rouchon, P. (2002). On the control of quantum oscillators. Technical Report A/320. École des Mines de Paris, Centre Automatique et Systèmes.

Turinici,

G. (2000a). Analyse de méthodes numériques de simulation et contrôle en chimie quantique. $\mathrm{PhD}$ thesis. Université Paris Sud. http://www-rocq.inria.fr/Gabriel. Turinici $/$.

Turinici, G. (2000b). Controllable quantities for bilinear quantum systems. In: Proceedings of the 39th IEEE Conference on Decision and Control. pp. 1364-1369.

Vettori, P. (2002). On the convergence of a feedback control strategy for multilevel quantum systems. In: Fifteenth International Symposium on Mathematical Theory of Networks and Systems (MTNS). 